\title{
Weak Galerkin Finite Element Methods for Darcy Flow: Anisotropy and Heterogeneity
}

\author{
Guang Lin ${ }^{\mathrm{a}, \mathrm{b}, *}$, Jiangguo $\mathrm{Liu}^{\mathrm{c}}, \operatorname{Lin} \mathrm{Mu}^{\mathrm{d}}$, Xiu $\mathrm{Ye}^{\mathrm{e}}$ \\ a Department of Mathematics and School of Mechanical Engineering, Purdue University, \\ West Lafayette, IN 47907, USA \\ ${ }^{b}$ Computional Mathematics, Pacific Northwest National Lab, 902 Battelle Blvd., Box 999, \\ Richland, WA 99352, USA \\ c Department of Mathematics, Colorado State University, Fort Collins, CO 80523-1874, \\ USA \\ ${ }^{d}$ Department of Mathematics, Michigan State University, East Lansing, MI 48824, USA \\ e Department of Mathematics, University of Arkansas at Little Rock, Little Rock, AR \\ 72204, USA
}

\begin{abstract}
This paper presents a family of weak Galerkin finite element methods (WGFEMs) for Darcy flow computation. The WGFEMs are new numerical methods that rely on the novel concept of discrete weak gradients. The WGFEMs solve for pressure unknowns both in element interiors and on the mesh skeleton. The numerical velocity is then obtained from the discrete weak gradient of the numerical pressure. The new methods are quite different than many existing numerical methods in that they are locally conservative by design, the resulting discrete linear systems are symmetric and positive-definite, and there is no need for tuning problem-dependent penalty factors. We test the WGFEMs on benchmark problems to demonstrate the strong potential of these new methods in handling strong anisotropy and heterogeneity in Darcy flow.
\end{abstract}

Keywords: anisotropy, Darcy flow, heterogeneity, porous media, weak Galerkin

\section{Introduction}

For convenience of presentation, we concentrate on the two-dimensional Darcy equation, which is usually formulated as

$$
\left\{\begin{array}{l}
\nabla \cdot(-\mathbf{K} \nabla p) \equiv \nabla \cdot \mathbf{u}=f, \quad \mathbf{x} \in \Omega \\
p=p_{D}, \quad \mathbf{x} \in \Gamma^{D} \\
\mathbf{u} \cdot \mathbf{n}=u_{N}, \quad \mathbf{x} \in \Gamma^{N}
\end{array}\right.
$$

* Corresponding author.

Email address: guanglin@purdue.edu (Guang Lin) 
where $\Omega \subset \mathbb{R}^{2}$ is a bounded polygonal domain, $p$ the unknown pressure, $\mathbf{u}$ the Darcy velocity, $\mathbf{K}$ a permeability tensor that is uniformly symmetric positivedefinite, $f$ a source term, $p_{D}, u_{N}$ are respectively Dirichlet and Neumann boundary data, $\mathbf{n}$ the unit outward normal vector on $\partial \Omega$, which has a nonoverlapping decomposition $\Gamma^{D} \cup \Gamma^{N}$. When $\Gamma^{D} \neq \emptyset$, the problem has a unique solution.

Here is a list of commonly used finite element methods for solving the Darcy equation, or more generally, elliptic boundary value problems.

- Continuous Galerkin (CG) FEMs [7]. There are different opinions on whether CGFEMs are not locally conservative [21]. CGFEMs generally have smaller numbers of unknowns. CGFEMs assume continuous (polynomial) approximants and are not flexible for certain problems that have solutions with low regularity.

- Discontinuous Galerkin (DG) FEMs [2] (and the references therein). DGFEMs utilize discontinuous shape functions that exhibit great flexibility in handling complicated domain geometry and problems with low regularity. DGFEMs are parallelizable by design. But for the symmetric formulations, one has to choose sufficiently large penalty factors to ensure stability of the numerical methods [18]. Choosing appropriate penalty factors is problem-dependent and hence a drawback in applications of DGFEMs.

- DGFEMs with weak overpenalization (WOP) [6]. The inconvenience of choosing penalty factors motivates the weakly over-penalized DGFEMs, which avoid choosing penalty factors by means of weak over-penalization. However, these methods result in large condition numbers, even though simple block-diagonal preconditioners are available [6].

- Mixed FEMs [9]. The mixed methods solve for the pressure and Darcy velocity simultaneously. But it is well known that the mixed FEMs result in saddle-point problems, whose solvers are more complicated than those for definite systems [5].

- Hybridized FEMs [13] (and the references therein). Hybridization introduces new unknowns (Lagrangian multipliers) and relax the continuity constraints across element interfaces. Hybridization proliferates (conceptually) the numbers of unknowns, although degrees of freedom are actually smaller after certain variables are eliminated.

The weak Galerkin finite element methods introduced in [34] adopt a completely different approach. They rely on novel concepts such as weak functions, the weak gradient operator, discrete weak functions, and discrete weak gradients. As is well known, the variational formulation of a second order elliptic problem relies on the duality of the classical gradient operator. Locality of a finite element space implies relative independence of elementwise shape functions and local conservation. However, shape functions in element interiors could be related to their values on the element interfaces through integration by parts. 
The weak gradient operator characterizes exactly this connection, see Equation (3) in Section 2. Discrete weak gradient operators inherit the connection for finite dimensional (Galerkin type) polynomial approximation subspaces, see Equation (5) in Section 2.

The Darcy's law has its fundamental importance in flow and transport in porous media $[12,15,16,17,19,23,24,27]$. Although Darcy's law can be essentially treated as an elliptic problem, the quantities of interest are pressure and velocity (flux) and the focus is placed on flow features. In this paper, we investigate the performance of the novel WGFEMs on handling the numerical difficulties brought up by anisotropy and heterogeneity in permeability.

The rest of this paper is organized as follows. In Section 2, we explain a line of new concepts such as weak functions, weak gradients, discrete weak functions, and discrete weak gradients. Based on these, we develop weak Galerkin finite element methods for Darcy flow. Section 3 addresses implementation issues of the WGFEMs. Section 4 briefly reviews other existing numerical methods for Darcy flow. Section 5 presents numerical simulations of the WGFEMs on several benchmark problems for Darcy flow. Section 6 concludes the paper with some remarks.

\section{WGFEMs for Darcy Flow}

The weak Galerkin finite element methods were first introduced in [34] by using a weakly defined gradient operator over discontinuous functions. The concept of weak gradient and its approximations result in discrete weak gradients, which will play an important role in the WGFEMs for Darcy flow.

\subsection{Weak Functions and Discrete Weak Gradients}

Let $E$ be a triangular or rectangular element with interior $E^{\circ}$ and boundary $E^{\partial}:=\partial E$.

A weak function on $E$ refers to a pair of scalar-valued functions $v=$ $\left\{v^{\circ}, v^{\partial}\right\}$ such that $v^{\circ} \in L_{2}\left(E^{\circ}\right)$ and $v^{\partial} \in H^{\frac{1}{2}}(\partial E)$. Here $v^{\circ}$ can be understood as the value of $v$ in the interior of $E$, whereas $v^{\partial}$ is the value of $v$ on the boundary of $E$. Note that $v^{\partial}$ may not necessarily be the trace of $v^{\circ}$, should a trace be defined. We denote by $W(E)$ the space of all weak functions on $E$ :

$$
W(E)=\left\{v=\left\{v^{\circ}, v^{\partial}\right\}: v^{\circ} \in L_{2}\left(E^{\circ}\right), v^{\partial} \in H^{\frac{1}{2}}(\partial E)\right\} .
$$

For any weak function $v \in W(E)$, its weak gradient $\nabla_{w} v$ is defined (interpreted) as a linear functional on $H(\operatorname{div}, E)$ :

$$
\left(\nabla_{w} v, \mathbf{w}\right)=\int_{\partial E} v^{\partial}(\mathbf{w} \cdot \mathbf{n}) d s-\int_{E} v^{\circ}(\nabla \cdot \mathbf{w}) d E, \quad \forall \mathbf{w} \in H(\operatorname{div}, E) .
$$

Let $P^{l}\left(E^{\circ}\right)$ be the space of polynomials on $E^{\circ}$ with degree at most $l \geq 0$ and $P^{m}(\partial E)$ be the space of polynomials on $\partial E$ with degree at most $m \geq 0$. 
A discrete weak function is a weak function $v=\left\{v^{\circ}, v^{\partial}\right\}$ such that $v^{\circ} \in$ $P^{l}\left(E^{\circ}\right)$ and $v^{\partial} \in P^{m}(\partial E)$. A discrete weak function space on $E$ is defined as

$$
W(E, l, m)=\left\{v=\left\{v^{\circ}, v^{\partial}\right\}: v^{\circ} \in P^{l}\left(E^{\circ}\right), v^{\partial} \in P^{m}(\partial E)\right\} .
$$

To introduce a discrete weak gradient operator, we need a polynomial vector space. Let $n$ be any nonnegative integer and $P^{n}(E)^{2}$ be the space of vectorvalued polynomials with degree at most $n$. Let $V(E, n)$ be a subspace of $P^{n}(E)^{2}$. For any $v \in W(E, l, m)$, its discrete weak gradient is so defined that $\nabla_{w, d} v \in$ $V(E, n)$ is the unique solution of

$$
\int_{E} \nabla_{w, d} v \cdot \mathbf{w} d E=\int_{\partial E} v^{\partial}(\mathbf{w} \cdot \mathbf{n}) d s-\int_{E} v^{\circ}(\nabla \cdot \mathbf{w}) d E, \quad \forall \mathbf{w} \in V(E, n) .
$$

It can be observed that the discrete weak gradient operator $\nabla_{w, d}$ is a Galerkintype approximation of the weak gradient operator using a polynomial vector space $V(E, n)$.

For later use, we define a projection operator $Q_{h}=\left(Q_{h}^{\circ}, Q_{h}^{\partial}\right)$ into a discrete weak function space, where $Q_{h}^{\circ}$ is the $L_{2}$-projection into the function space on $E^{\circ}$ and $Q_{h}^{\partial}$ is the $L_{2}$-projection into the function space on $E^{\partial}$.

\subsection{Weak Finite Element Spaces}

Let $\mathcal{E}_{h}$ be a quasi-uniform triangular or rectangular partition on $\Omega, \Gamma_{h}$ be the set of all element interfaces (edges), $\Gamma_{h}^{I}$ the set of all interior edges, and $\Gamma_{h}^{b}$ the set of the edges on the domain boundary $\partial \Omega$.

Patching all elementwise discrete weak function spaces over the mesh $\mathcal{E}_{h}$, we obtain a weak finite element space as

$$
S_{h}\left(\mathcal{E}_{h}, l, m\right)=\left\{v=\left\{v^{\circ}, v^{\partial}\right\}:\left.v\right|_{E} \in W(E, l, m) \forall E \in \mathcal{E}_{h}\right\} .
$$

Furthermore, we define

$$
S_{h}^{0}\left(\mathcal{E}_{h}, l, m\right)=\left\{v=\left\{v^{\circ}, v^{\partial}\right\} \in S_{h}\left(\mathcal{E}_{h}, l, m\right),\left.v^{\partial}\right|_{\partial E \cap \Gamma^{D}}=0 \forall E \in \mathcal{E}_{h}\right\} .
$$

Examples of weak finite element spaces include those consisting of constant or linear shape functions, which are of practical interest due to their simplicity in implementation.

- The triangular space (WGRT0) consists of weak shape functions that are constants on elements in a triangular mesh and constants on edges in the mesh. Their discrete weak gradients are in the Raviart-Thomas space $R T_{0}(E)$ for each triangle $E$ in the mesh. A full name would be $\left(P_{0}, P_{0}, R T_{0}\right)$.

- The rectangular space (WGRT0) consists of weak shape functions that are constants on elements in a rectangular mesh and constants on edges in the mesh. Their discrete weak gradients are in the Raviart-Thomas space $R T_{[0]}(E)$ for each rectangle $E$ in the mesh. The full name is $\left(Q_{0}, P_{0}, R T_{[0]}\right)$. 
Here are some other weak Galerkin finite element spaces.

- The triangular $\left(P_{l}, P_{l}, R T_{l}\right)$ space for any integer $l \geq 1$, namely, $S_{h}(l, l)$ together with $V(E, l+1)=R T_{l}(E)$ for any integer $l \geq 1$.

- The triangular $\left(P_{l}, P_{l+1}, P_{l+l}^{2}\right)$ space for any integer $l \geq 0$, in other words, $S_{h}(l, l+1)$ together with $V(E, l+1)=P^{l+1}(E)^{2}$ for any integer $l \geq 0$.

- The rectangular $\left(Q_{l}, P_{l}, R T_{[l]}\right)$ space for any integer $l \geq 1$, namely, $S_{h}(l, l)$ together with $V(E, l+1)=R T_{[l]}(E)$ for any integer $l \geq 1$.

Although a WG finite element space can be constructed for any combination of $l, m, n$, good approximate solutions to partial differential equations could be produced only for certain combinations of $l, m, n$. For the WGFEMs to work well, two prominent properties must be satisfied by a discrete weak gradient operator [34].

- Property P1 (Still Property). Let $v \in S_{h}(l, m)$ and $E \in \mathcal{E}_{h}$. If $\nabla_{w, d} v=0$ on $E$, then $v^{\circ}=$ const on $E^{\circ}$ and $v^{\partial}=$ const on $\partial E$.

- Property P2 (Approximation Capacity). For $u \in H^{s}(\Omega), s \geq 1$, let $Q_{h} u \in S_{h}(l, m)$ be an interpolation or projection of $u$. Then the discrete weak gradient of $Q_{h} u$ should be a good approximation of $\nabla u$.

\subsection{Weak Galerkin Finite Element Schemes for Darcy Flow}

Let $\mathcal{E}_{h}$ be a triangular or rectangular mesh. It is known from [34] that the following WG finite elements have the aforementioned two properties:

- For $k \geq 0,\left(P_{k}, P_{k}, R T_{k}\right)$ on triangles;

- For $k \geq 0,\left(Q_{k}, P_{k}, R T_{[k]}\right)$ on rectangles.

These elements will be employed to establish numerical schemes for solving the Darcy equation.

Based on the variational formulation of $(1)$ on $H^{1}(\Omega)$, we take a two-step procedure described below [34]:

(i) Approximating $H^{1}(\Omega)$ by a space of discrete weak functions which are properly constructed on the mesh $\mathcal{E}_{h}$;

(ii) Approximating the classical gradient operator $\nabla$ by a discrete weak gradient operator $\nabla_{w, d}$ that acts on the space of discrete weak functions defined above.

Then we define bilinear and linear forms on a WG finite element space as

$$
\mathcal{A}_{h}\left(p_{h}, q\right):=\sum_{E \in \mathcal{E}_{h}} \int_{E} \mathbf{K} \nabla_{w, d} p_{h} \cdot \nabla_{w, d} q d E,
$$




$$
\mathcal{F}(q):=\sum_{E \in \mathcal{E}_{h}} \int_{E} f q^{\circ} d E-\sum_{\gamma \in \Gamma_{h}^{N}} \int_{\gamma} u_{N} q d s .
$$

Now a Weak Galerkin Finite Element Scheme for the Darcy equation (1) reads as: Seek $p_{h}=\left\{p_{h}^{\circ}, p_{h}^{\partial}\right\} \in S_{h}(l, m)$ such that $\left.p_{h}^{\partial}\right|_{\Gamma^{D}}=Q_{h}^{\partial} p_{D}$ and

$$
\mathcal{A}_{h}\left(p_{h}, q\right)=\mathcal{F}(q), \quad \forall q=\left\{q^{\circ}, q^{\partial}\right\} \in S_{h}^{0}(l, m) .
$$

Let $p_{h}=\left\{p_{h}^{\circ}, p_{h}^{\partial}\right\}$ be the numerical pressure obtained from solving (10), then we define

$$
\mathbf{u}_{h}=R_{h}\left(-\mathbf{K} \nabla_{w, d} p_{h}\right)
$$

as the numerical velocity, where $R_{h}$ is the local $L_{2}$ projection onto $V(E, n)$.

Next we state three theorems regarding the accuracy and properties of the numerical pressure and velocity obtained from using the WGFEMs with elements $\left(P_{l}, P_{l}, R T_{l}\right), l=0,1$ on triangular meshes or $\left(Q_{l}, P_{l}, R T_{[l]}\right), l=0,1$ on rectangular meshes.

Theorem 1. Let $(p, \mathbf{u})$ be the exact solutions of (1). If the solutions are sufficiently smooth, i.e., there is an integer $m \geq 2$ such that $p \in H^{m}(\Omega)$ and $\mathbf{u} \in H^{m-1}(\Omega)^{2}$, then

$$
\left\|p-p_{h}^{\circ}\right\|_{L^{2}(\Omega)}=\mathcal{O}\left(h^{r_{0}}\right), \quad\left\|Q_{h}^{\circ} p-p_{h}^{\circ}\right\|_{L^{2}(\Omega)}=\mathcal{O}\left(h^{r_{0}+1}\right), \quad\left\|\mathbf{u}-\mathbf{u}_{h}\right\|_{L^{2}(\Omega)}=\mathcal{O}\left(h^{r_{1}}\right),
$$

where $r_{0}=\min (m, k), r_{1}=\min (m-1, k)$ and $k$ is an integer related to the WG finite elements being used. For $\left(P_{0}, P_{0}, R T_{0}\right)$ and $\left(Q_{0}, P_{0}, R T_{[0]}\right)$, we have $k=1$; for $\left(P_{1}, P_{1}, R T_{1}\right)$ and $\left(Q_{1}, P_{1}, R T_{[1]}\right)$, we have $k=2$. If the solutions have low regularity, that is, $p \in H^{1+s}(\Omega), \mathbf{u} \in H^{s}(\Omega)^{2}$ for some $s \in\left(\frac{1}{2}, 1\right)$, then

$$
\left\|p-p_{h}^{\circ}\right\|_{L^{2}(\Omega)}=\mathcal{O}\left(h^{r}\right), \quad\left\|Q_{h}^{\circ} p-p_{h}^{\circ}\right\|_{L^{2}(\Omega)}=\mathcal{O}\left(h^{2 r}\right), \quad\left\|\mathbf{u}-\mathbf{u}_{h}\right\|_{L^{2}(\Omega)}=\mathcal{O}\left(h^{r}\right),
$$

where $r=\min (s, k)$ but $k$ is the same as above.

Theorem 2. (Local mass conservation.) For any element $E \in \mathcal{E}_{h}$, there holds

$$
\int_{\partial E} \mathbf{u}_{h} \cdot \mathbf{n} d s=\int_{E} f d E .
$$

Theorem 3. (Normal flux continuity.) Let $\gamma \in \Gamma_{h}^{I}$ be an interior edge shared by two elements $E_{1}, E_{2}, \mathbf{n}_{i}$ be the unit outer normal of element $E_{i}(i=$ $1,2)$ on $\gamma$, and $\mathbf{u}_{h}^{(i)}$ be the numerical velocity on element $E_{i}(i=1,2)$. Then

$$
\int_{\gamma} \mathbf{u}_{h}^{(1)} \cdot \mathbf{n}_{1} d s=\int_{\gamma} \mathbf{u}_{h}^{(2)} \cdot \mathbf{n}_{2} d s .
$$

Remark 2.1. In applications for Darcy flow, where the permeability $\mathbf{K}$ is usually piecewise constant, the exact pressure $p$ is no longer in $H^{2}(\Omega)$. Instead, $p \in H^{1+s}(\Omega)(0<s<1)$ and $s$ is inversely proportional to the magnitude of 
jumps in $\mathbf{K}$. Theorem 1 indicates that the accuracy of the numerical pressure and velocity is restricted by the regularity of the problem, which explains why the lowest order WG finite elements are preferred for solving the Darcy equation.

Remark 2.2. A proof of Theorem 1 for the smooth case can be derived from the analysis in [34]. A proof of Theorem 1 for the low regularity case needs some techniques similar to those used in [26]. Proofs of Theorems 2 and 3 can be found in [34].

\section{Implementation of WGFEMs for Darcy Flow}

For WGFEM formulations, it is assumed that permeability is in $L^{\infty}$. For implementation, it is convenient to assume the permeability is a constant scalar or a constant SPD matrix on each element of a given mesh. In general, permeability could vary spatially within a cell/element. If the changes are not dramatic, then an average of the permeability scalar/matrix could be used. For dramatics changes, multiscale methods could be used [17].

For convenience, we assume the meshes are either triangular or rectangular and they satisfy the shape-regularity assumption [7]. There are no hangingnodes in the meshes.

There is a good list of admissible choices for WG finite elements, as discussed in the previous section and in [34]. In this section, we consider mainly the lowest order WG finite elements $\left(P_{0}, P_{0}, R T_{0}\right),\left(Q_{0}, P_{0}, R T_{[0]}\right)$, due to the easiness in implementation and practical usefulness in Darcy flow computation. Triangular elements $\left(P_{1}, P_{1}, R T_{1}\right)$ will also be discussed to show how higher order elements are used.

An important part in WGFEM implementations is the calculation of the discrete weak gradients of the chosen WG basis functions. These are prescribed by Equation (5). Since all these discrete weak gradients are linear combinations of the vector-valued basis functions of $V(E, n)$, e.g., $R T_{0}, R T_{[0]}$ (see Section 2.1), one needs to solve small-size linear systems depicted by Equation (5). Thus the Gram matrix of these basis functions for $V(E, n)$ is needed. It will also be convenient if the Gram matrix can be simply inverted.

\subsection{WG Basis Functions and Their Discrete Weak Gradients}

\subsubsection{Triangular $\left(P_{0}, P_{0}, R T_{0}\right)$ Elements}

Let $T$ be a triangular element with vertices $P_{i}\left(x_{i}, y_{i}\right)(i=1,2,3)$ oriented counterclockwise. Let $|T|$ be the triangle area, $\left(x_{c}, y_{c}\right)$ the center of the element, $e_{i}(i=1,2,3)$ the edge opposite to vertex $P_{i}$, and $\left|e_{i}\right|$ edge length.

There is only one WG basis function for $P_{0}\left(T^{\circ}\right)$, which takes constant value 1 in the triangle interior, expressed as

$$
\left.\phi^{\circ}\right|_{T^{\circ}}=1 .
$$


There are three WG basis functions for $P_{0}(\partial T)$ that takes constant value 1 on one edge and 0 on the other two edges. In other words, we have

$$
\left.\phi_{i}^{\partial}\right|_{e_{j}}=\delta_{i j}, \quad 1 \leq i, j \leq 3,
$$

where $\delta_{i j}$ is the Kronecker symbol.

With the previously defined local WG basis functions, the global WG basis functions will be simply the "gluing-together" of the local basis functions.

Before the discrete weak gradients of the above 4 WG basis functions can be calculated, a set of basis functions for $R T_{0}(T)$ need to be specified. These could be the edge-oriented basis functions [3, 31] or normalized basis functions [25]:

$$
\left[\begin{array}{l}
1 \\
0
\end{array}\right], \quad\left[\begin{array}{l}
0 \\
1
\end{array}\right], \quad\left[\begin{array}{l}
X \\
Y
\end{array}\right],
$$

where $X=x-x_{c}, Y=y-y_{c}$. For convenience, we name them as $\mathbf{w}_{1}, \mathbf{w}_{2}, \mathbf{w}_{3}$. As can be seen later, one advantage of the latter is that the Gram matrix will be diagonal and hence trivially inverted.

The Gram matrix of the above normalized basis is simply diagonal:

$$
G M=\operatorname{diag}(|T|,|T|, S),
$$

where

$$
\begin{aligned}
S & =\frac{|T|}{36}\left(\left(x_{1}-x_{2}\right)^{2}+\left(x_{2}-x_{3}\right)^{2}+\left(x_{3}-x_{1}\right)^{2}\right. \\
& \left.+\left(y_{1}-y_{2}\right)^{2}+\left(y_{2}-y_{3}\right)^{2}+\left(y_{3}-y_{1}\right)^{2}\right) .
\end{aligned}
$$

Next, the definition formula (5) is employed to calculate the discrete weak gradients of $\phi^{\circ}, \phi_{i}^{\partial}(i=1,2,3)$. Since the Gram matrix of the above normalized basis is diagonal, one can easily obtain these discrete weak gradients as follows

$$
\begin{aligned}
\partial_{w, d} \phi^{\circ} & =\frac{|T|}{S}\left(0 \mathbf{w}_{1}+0 \mathbf{w}_{2}+(-2) \mathbf{w}_{3}\right), \\
\partial_{w, d} \phi_{1}^{\partial} & =\frac{y_{3}-y_{2}}{|T|} \mathbf{w}_{1}+\frac{x_{2}-x_{3}}{|T|} \mathbf{w}_{2}+\frac{2|T|}{3 S} \mathbf{w}_{3}, \\
\partial_{w, d} \phi_{2}^{\partial} & =\frac{y_{1}-y_{3}}{|T|} \mathbf{w}_{1}+\frac{x_{3}-x_{1}}{|T|} \mathbf{w}_{2}+\frac{2|T|}{3 S} \mathbf{w}_{3}, \\
\partial_{w, d} \phi_{3}^{\partial} & =\frac{y_{2}-y_{1}}{|T|} \mathbf{w}_{1}+\frac{x_{1}-x_{2}}{|T|} \mathbf{w}_{2}+\frac{2|T|}{3 S} \mathbf{w}_{3} .
\end{aligned}
$$

\subsubsection{Rectangular $\left(Q_{0}, P_{0}, R T_{[0]}\right)$ Elements}

Let $E=\left[x_{1}, x_{2}\right] \times\left[y_{1}, y_{2}\right]$ be a typical rectangular element, $|E|$ the element area, and $\left(x_{c}, y_{c}\right)$ the element center.

It is known that $\operatorname{dim}\left(R T_{[0]}(E)\right)=4$. Again, we choose normalized basis functions as follows

$$
\mathbf{w}_{1}=\left[\begin{array}{l}
1 \\
0
\end{array}\right], \quad \mathbf{w}_{2}=\left[\begin{array}{l}
0 \\
1
\end{array}\right], \quad \mathbf{w}_{3}=\left[\begin{array}{c}
X \\
0
\end{array}\right], \quad \mathbf{w}_{4}=\left[\begin{array}{c}
0 \\
Y
\end{array}\right],
$$


where we have set $X=x-x_{c}, Y=y-y_{c}$ for convenience. The Gram matrix is again diagonal

$$
G M=\operatorname{diag}\left(|E|,|E|, \frac{1}{12}\left(x_{2}-x_{1}\right)^{2}|E|, \frac{1}{12}\left(y_{2}-y_{1}\right)^{2}|E|\right) .
$$

For WG, we have now one constant basis function in the element interior $E^{\circ}$ and one constant basis function on each edge of the rectangle. Their discrete weak gradients, actually the coefficients in the above $R T_{[0]}(E)$ basis, can be calculated by employing the definition formula (5). The results are

$$
\left[\begin{array}{c}
\nabla_{w, d} \phi^{\circ} \\
\nabla_{w, d} \phi_{1}^{\partial} \\
\nabla_{w, d} \phi_{2}^{\partial} \\
\nabla_{w, d} \phi_{3}^{\partial} \\
\nabla_{w, d} \phi_{4}^{\partial}
\end{array}\right]=\left[\begin{array}{cccc}
0 & 0 & \frac{-12}{\left(x_{2}-x_{1}\right)^{2}} & \frac{-12}{\left(y_{2}-y_{1}\right)^{2}} \\
0 & \frac{-1}{y_{2}-y_{1}} & 0 & \frac{6}{\left(y_{2}-y_{1}\right)^{2}} \\
\frac{1}{x_{2}-x_{1}} & 0 & \frac{6}{\left(x_{2}-x_{1}\right)^{2}} & 0 \\
0 & \frac{1}{y_{2}-y_{1}} & 0 & \frac{6}{\left(y_{2}-y_{1}\right)^{2}} \\
\frac{-1}{x_{2}-x_{1}} & 0 & \frac{6}{\left(x_{2}-x_{1}\right)^{2}} & 0
\end{array}\right]\left[\begin{array}{l}
\mathbf{w}_{1} \\
\mathbf{w}_{2} \\
\mathbf{w}_{3} \\
\mathbf{w}_{4}
\end{array}\right]
$$

\subsubsection{Triangular $\left(P_{1}, P_{1}, R T_{1}\right)$ Elements}

Let $T$ be a typical triangular element with center $\left(x_{c}, y_{c}\right)$. Again for convenience, we set $X=x-x_{c}, Y=y-y_{c}$.

Notice that $\operatorname{dim}\left(R T_{1}(T)\right)=8$, as shown in Figure 2. We could choose the following eight functions as its basis:

$$
\left[\begin{array}{l}
1 \\
0
\end{array}\right],\left[\begin{array}{c}
X \\
0
\end{array}\right],\left[\begin{array}{l}
Y \\
0
\end{array}\right],\left[\begin{array}{l}
0 \\
1
\end{array}\right],\left[\begin{array}{l}
0 \\
X
\end{array}\right],\left[\begin{array}{c}
0 \\
Y
\end{array}\right],\left[\begin{array}{c}
X^{2} \\
X Y
\end{array}\right],\left[\begin{array}{c}
X Y \\
Y^{2}
\end{array}\right] .
$$

The Gram matrix is $8 \times 8$ and symmetric positive-definite.

For a WG triangular $\left(P_{1}, P_{1}, R T_{1}\right)$ element, $P_{1}\left(T^{\circ}\right)$ has 3 basis functions that can be chosen as $1, X, Y ; P_{1}\left(T^{\partial}\right)$ has 2 basis functions for each edge. These can be chosen as $1, t$, where $t \in[-1,1]$ is the parameter for each edge. For example, the first edge has parametrization $x=\left(x_{3}+x_{2}\right) / 2+t\left(x_{3}-x_{2}\right) / 2, y=$ $\left(y_{3}+y_{2}\right) / 2+t\left(y_{3}-y_{2}\right) / 2$.

The discrete weak gradients of the above nine WG basis functions are calculated using the definition formula (5). So nine $8 \times 8$ linear systems need to be solved to obtain the linear combination coefficients of $\nabla_{w, d} \phi$ in terms of the eight vector basis functions in (16).

\subsection{Assembling and Solving of the Global Discrete Linear System}

The previously computed discrete weak gradients of the WG basis functions will be used in calculations of the local stiffness matrices. Based on Formula (8), all these local stiffness matrices are assembled into a global coefficient matrix. For convenience and efficiency of assembly, we separate WGFEM unknowns into two groups: those in element interiors and those on edges. Then the global matrix will have a clear block structure

$$
A=\left[\begin{array}{cc}
A_{e e} & A_{e g} \\
A_{g e} & A_{g g}
\end{array}\right] \text {. }
$$


Here $A_{e e}$ reflects the interaction of individual elements with themselves. For triangular $\left(P_{0}, P_{0}, R T_{0}\right)$ elements or rectangular $\left(Q_{0}, P_{0}, R T_{[0]}\right)$ elements, this means $A_{e e}$ is simply a diagonal matrix with size being the number of elements in a given mesh. $A_{e g}$ reflects the interaction of individual elements with their 3 or 4 edges. $A_{g e}$ is the transpose of $A_{e g}$. $A_{g g}$ reflects the interaction of all edges.

Therefore, for WG $\left(P_{0}, P_{0}, R T_{0}\right)$ on triangular meshes, the bandwidth of the global coefficient matrix is 7 , because the unknown in an element interior interacts with itself and the three edge unknowns, whereas the unknown on an edge interacts with the 2 unknowns in adjacent element interior plus 5 unknowns on adjacent edges (including itself). Similarly, for WG $\left(Q_{0}, P_{0}, R T_{[0]}\right)$ on rectangular meshes, the bandwith of the global coefficient matrix is 9 .

The global linear system is symmetric positive-definite and has a condition number $\mathcal{O}\left(h^{-2}\right)$ (where $h$ is the mesh size), so that it can be readily solved by any standard direct or iterative solver, e.g., the conjugate gradient method.

The aforementioned block structure in the global stiffness matrix also indicates that Schur complement could be used for solving the global linear system.

\subsection{Computing the Numerical Velocity}

After solving the discrete linear system (10) over a given mesh, a numerical pressure $p_{h}$ is obtained in element interiors and on the mesh skeleton. Its discrete weak gradient $\nabla_{w, d} p_{h}$ is then calculated on each element. This will be a linear combination of the discrete weak gradients of the WG basis functions. It is in the local $R T_{0}, R T_{[0]}, R T_{1}$, or $R T_{[1]}$ spaces, as specified when the WG finite element is chosen.

The next step will be the local $L_{2}$ projection of the quantity $-\mathbf{K} \nabla_{w, d} p_{h}$ into the aforementioned local $H(\mathrm{div})$-conforming subspace on each element:

$$
\mathbf{u}_{h}=R_{h}\left(-\mathbf{K} \nabla_{w, d} p_{h}\right) .
$$

This involves solving a small-size linear system on each element and can be done independently (or in parallel).

However, if the permeability is a piecewise constant scalar on the given mesh, that is, $\mathbf{K}=K_{E} \mathbf{I}_{2}$, where $K_{E}$ takes a constant scalar value on each element and $\mathbf{I}_{2}$ is the $2 \times 2$ identity matrix, then there is no need for the above local $L_{2}$ projection. This brings in convenience for practical applications.

Finally, for any edge $\gamma$ of an element $E$, the edge flux $\int_{\gamma} \mathbf{u}_{h} \cdot \mathbf{n}_{\gamma} d s\left(\mathbf{n}_{\gamma}\right.$ is the unit outer normal) is calculated.

\section{Other Numerical Methods for Darcy Flow}

In this section, we briefly review other commonly used numerical methods for Darcy flow. Due to limitation of space, we discuss mainly three types of methods: the cell-centered finite difference method [19], the classical mixed finite element methods $[1,3,9,28]$, and some control-volume finite volume methods $[15,16,30]$. 


\subsection{The Cell-Centered Finite Difference Method}

The cell-centered finite difference method is used by the vast majority of working codes in the petroleum industry for solving the Darcy equation [19]. It is based on physical considerations (the integral form of mass conservation) and discretization of gradients using finite differences. It also uses harmonic averages of permeability over adjacent cells. In this approach, the unknowns are the pressure averages on the cells (elements). Below is a brief account of this method when the permeability is a diagonal tensor.

Let $\Omega=[a, b] \times[c, d]$ be a two-dimensional rectangular domain. Let $a=$ $x_{\frac{1}{2}}<x_{\frac{3}{2}}<\cdots<x_{i-\frac{1}{2}}<x_{i+\frac{1}{2}}<\cdots<x_{m+\frac{1}{2}}=b$ be a partion in the $x$ direction. Similarly, Let $c=y_{\frac{1}{2}}<y_{\frac{3}{2}}<\cdots<y_{j-\frac{1}{2}}<y_{j+\frac{1}{2}}<\cdots<y_{n+\frac{1}{2}}=d$ be a partition in the $y$-direction. See Figure 1 . We set

$$
\begin{aligned}
& x_{i}=\frac{1}{2}\left(x_{i-\frac{1}{2}}+x_{i+\frac{1}{2}}\right), \quad i=1,2, \ldots, m ; \\
& y_{j}=\frac{1}{2}\left(y_{j-\frac{1}{2}}+y_{j+\frac{1}{2}}\right), \quad j=1,2, \ldots, n ;
\end{aligned}
$$

so that $\left(x_{i}, y_{j}\right)$ is the center of cell/element $E_{i, j}$. The $x, y$-direction lengths of $E_{i, j}$ are denoted respectively as $\Delta x_{i}, \Delta y_{j}$.

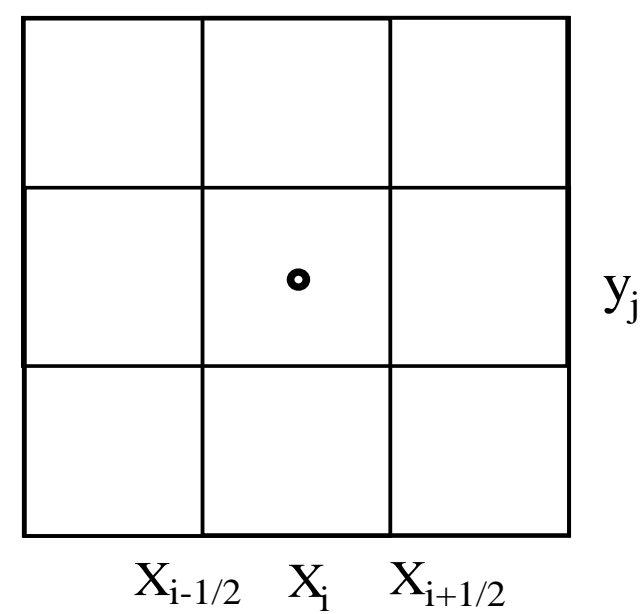

Figure 1: A stencil for the cell-centered finite difference method.

Consider a typical cell $E_{i, j}$. By the conservation law and the Divergence Theorem, we have from (1) that

$$
\int_{E_{i j}} f d x d y=\int_{E_{i j}} \nabla(-\mathbf{K} \nabla p) d x d y=\int_{\partial E_{i j}}(-\mathbf{K} \nabla p \cdot \mathbf{n}) d s .
$$

We need permeability on the cell edges. These could be taken as harmonic averages

$$
K_{i+\frac{1}{2}, j}=\frac{K_{i, j} K_{i+1, j}}{K_{i, j}+K_{i+1, j}}
$$


assuming $\mathbf{K}=K \mathbf{I}_{2}$ is a scalar matrix. Here $\mathbf{I}_{2}$ is the order 2 identity matrix. Similar treatments apply to $K_{i-\frac{1}{2}, j}, K_{i, j+\frac{1}{2}}, K_{i, j-\frac{1}{2}}$. For an interior element $E_{i, j}$, the outward normal flux on its right edge is approximated as

$$
\int_{\partial_{\text {right }} E_{i, j}}(-\mathbf{K} \nabla p \cdot \mathbf{n}) d s \approx-\frac{K_{i, j} K_{i+1, j}}{K_{i, j}+K_{i+1, j}} \frac{P_{i+1, j}-P_{i, j}}{\frac{\Delta x_{i}+\Delta x_{i+1}}{2}} \Delta y_{j} .
$$

Here $P_{i, j}, P_{i+1, j}$ are pressure unknowns on element $E_{i, j}, E_{i+1, j}$ respectively. Similarly, we have approximations for the other three flux terms. These approximants are substituted into Equation (17) to form a discrete linear system about the cell pressure unknowns $P_{i, j}, 1 \leq i \leq m, 1 \leq j \leq n$.

Described below is a recipe for treating Dirichlet and Neumann boundary conditions.

(i) When an edge of a cell is on the Neumann boundary of the domain, we use the prescribed outward normal flux in the Neumann boundary condition for the edge flux, this term is then moved to the right-hand side of the corresponding equation.

(ii) When an edge of a cell is on the Dirichlet boundary of the domain, we use the prescribed Dirichlet data to construct a finite difference. In this case, only the permeability on this cell is involved. For example, assume the left edge of $E_{1,1}$ is on the Dirichlet boundary, then we have $\mathbf{n}=(-1,0)$ and

$$
\int_{\partial_{\mathrm{left}} E_{1,1}}(-\mathbf{K} \nabla p \cdot \mathbf{n}) d s \approx K_{1,1} \frac{P_{1,1}-p_{D}\left(a, y_{1}\right)}{\frac{\Delta x_{1}}{2}} \Delta y_{1} .
$$

Equation (17) is then modified accordingly.

Remark 3.1. When a Neumann boundary condition is specified on the whole boundary of the domain, the pressure solution is unique up to an additive constant. Theoretically we require $\int_{\Omega} P_{h} d x d y=0$. In practice we could just set the numerical pressure in a chosen cell to zero or any specific value.

The discrete linear system resulting from the cell-centered finite difference scheme is definite and hence could be solved by any standard direct or iterative linear solver.

\subsection{The Mixed Finite Element Methods}

The classical mixed finite element methods (MFEMs) have been widely used for Darcy flow calculations. In MFEMs, the pressure and velocity are approximated simultaneously. This is based on rewriting the Darcy's law (1) as a system of first order partial differential equations about the pressure and velocity as follows $[9,12]$

$$
\left\{\begin{array}{l}
\mathbf{K}^{-1} \mathbf{u}+\nabla p=0, \quad \mathbf{x} \in \Omega \\
\nabla \cdot \mathbf{u}=f, \quad \mathbf{x} \in \Omega \\
\mathbf{u} \cdot \mathbf{n}=u_{N}, \quad \mathbf{x} \in \Gamma^{N} \\
p=p_{D}, \quad \mathbf{x} \in \Gamma^{D}
\end{array}\right.
$$


Let $\Omega \subset \mathbb{R}^{d}(d=2,3)$ be a bounded domain. We define

$$
\begin{aligned}
H(\operatorname{div} ; \Omega) & =\left\{\mathbf{v} \in L_{2}(\Omega)^{2}: \nabla \cdot \mathbf{v} \in L_{2}(\Omega)\right\}, \\
H_{0, N}(\operatorname{div} ; \Omega) & =\left\{\mathbf{v} \in H(\operatorname{div} ; \Omega): \mathbf{v} \cdot \mathbf{n}=0 \text { on } \Gamma^{N}\right\}, \\
H_{u_{N}, N}(\operatorname{div} ; \Omega) & =\left\{\mathbf{v} \in H(\operatorname{div} ; \Omega): \mathbf{v} \cdot \mathbf{n}=u_{N} \text { on } \Gamma^{N}\right\} .
\end{aligned}
$$

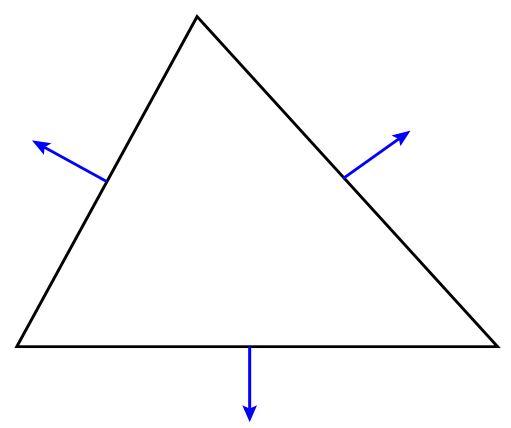

(a)

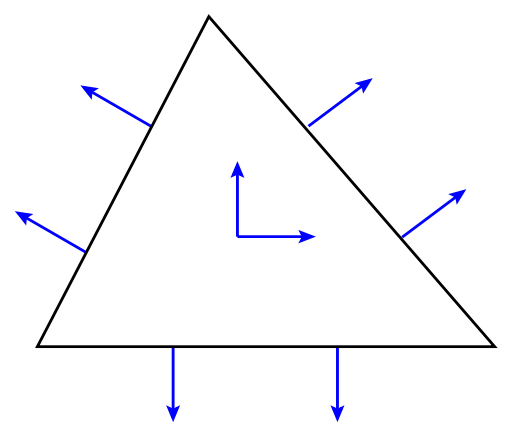

(b)

Figure 2: The Raviart-Thomas spaces on triangles: (a) $R T_{0}$ with 3 degrees of freedom (DOFs) for the flux/velocity; (b) $R T_{1}$ with 8 DOFs for the flux/velocity.

The mixed weak formulation for Darcy flow is: Seek $\mathbf{u} \in H_{u_{N}, N}(\operatorname{div}, \Omega)$ and $p \in L_{2}(\Omega)$ such that the following hold

$$
\begin{cases}\int_{\Omega}\left(\mathbf{K}^{-1} \mathbf{u}\right) \cdot \mathbf{v}-\int_{\Omega} p(\nabla \cdot \mathbf{v})=-\int_{\Gamma^{D}} p_{D} \mathbf{v} \cdot \mathbf{n} & \forall \mathbf{v} \in H_{0, N}(\operatorname{div}, \Omega) \\ -\int_{\Omega}(\nabla \cdot \mathbf{u}) q=-\int_{\Omega} f q & \forall q \in L_{2}(\Omega) .\end{cases}
$$

MFEMs are based on $H$ (div)-conforming finite elements and the inf-sup condition [9]. Denote $V=H(\operatorname{div} ; \Omega), W=L_{2}(\Omega)$. Let $V_{h} \subset V$ and $W_{h} \subset W$ be a pair of finite element spaces for velocity and pressure respectively. The inf-sup condition requires that

$$
\inf _{q \in W_{h}} \sup _{v \in V_{h}} \frac{B(v, q)}{\|v\|_{V}\|q\|_{W}}>0 .
$$

Let $U_{h}=V_{h} \cap H_{u_{N}, N}(\operatorname{div} ; \Omega)$ and $V_{h}^{0}=V_{h} \cap H_{0, N}(\operatorname{div} ; \Omega)$. A mixed finite element scheme can be stated as: Seek $\mathbf{u}_{h} \in U_{h}$ and $p_{h} \in W_{h}$ such that for any $\mathbf{v} \in V_{h}^{0}, q \in W_{h}$, the following hold

$$
\left\{\begin{array}{l}
\left(\mathbf{K}^{-1} \mathbf{u}_{h}, \mathbf{v}\right)_{\mathcal{E}_{h}}-\left(p_{h}, \nabla \cdot \mathbf{v}\right)_{\mathcal{E}_{h}}=-\left(p_{D}, \mathbf{v} \cdot \mathbf{n}\right)_{\Gamma_{h}^{D}}, \\
-\left(\nabla \cdot \mathbf{u}_{h}, q\right)_{\mathcal{E}_{h}}=-(f, q)_{\mathcal{E}_{h}}
\end{array}\right.
$$


It is well known that MFEMs result in indefinite discrete linear systems that require special solvers, e.g., the Uzawa algorithm [5, 9].

There are many element pairs that satisfy the inf-sup condition and hence could be used in the above mixed finite element scheme. The commonly used lowest order finite elements are $R T_{0}$ for triangular meshes (Fig. 2) and $R T_{[0]}$ for rectangular meshes. In each case, the pressure is approximated by constants on elements, the flux is approximated from the $R T_{0}$ or $R T_{[0]}$ space.

There are detailed accounts on implementing MFEM $\left(R T_{0}\right)$ in Matlab [3]. In $[1,28]$, the ideas of solving for pressure unknowns on both elements and edges were explored.

There are some interesting connection and differences between MFEM $R T_{0}$ and WG $\left(P_{0}, P_{0}, R T_{0}\right)$ on triangular meshes, similarly between MFEM $R T_{[0]}$ and WG $\left(Q_{0}, P_{0}, R T_{[0]}\right)$ on rectangular meshes.

(i) First note that in MFEM, the Neumann boundary condition is essential and the Dirichlet boundary condition is natural; whereas in WGFEMs, the Dirichlet condition is essential and the Neumann condition is natural.

(ii) The MFEM $R T_{0}$ on a triangular mesh uses $R T_{0}$ for approximating the flux $-\mathbf{K} \nabla p$; whereas the weak Galerkin $\left(P_{0}, P_{0}, R T_{0}\right)$ on a triangular mesh uses $R T_{0}$ for approximating the gradient $\nabla p$.

(iii) For both methods, the numerical flux is locally conservative on elements, the norm flux is continuous across element interfaces.

(iv) Both methods have the same number of unknowns. MFEM $R T_{0}$ has one pressure unknown on each element and one normal flux unknown on each edge; whereas WG $\left(P_{0}, P_{0}, R T_{0}\right)$ has one pressure unknown on each element and one pressure unknown on each edge.

(v) However, the major difference is that MFEMs result in saddle-point problems which are difficult to solve; whereas WGFEMs result in symmetric definite linear systems that are easier to solve.

\subsection{Finite Volume Methods and Mimetic Methods}

Finite volume methods form another important class of numerical methods for Darcy flow.

It is very interesting to see some similar ideas that were explored in the finite volume setting [30]:

- Use both cell-center and face-center scalar unknowns as opposed to just cell-center scalar unknowns;

- Define a modified gradient operator to approximate $-\mathbf{K} \nabla p$;

- The divergence and modified gradient operators are adjoint to one another;

- Define a discrete version of the divergence operator on a single cell; 
- Use the adjoint property to define a discrete version of the modified gradient operator on a single arbitrary cell.

While similarities between the ideas listed above and the concepts of weak gradients, discrete weak gradients, duality of divergence and gradient operators can be observed, the WGFEMs have a solid foundation on Sobolev spaces and systematic designs of various admissible finite elements.

The control volume methods in $[15,16]$ (see also the references therein) represent significant progresses in the development of finite volume methods for Darcy flow. These methods intend to address the challenging problems related to strong anisotropy in full tensor permeability field. These methods require only a single degree of freedom per control-volume. This family of methods include some tuning parameters. Based on well-designed quadratures addressing the anisotropy, these methods produce results with sharp resolution, with only minor or practically zero spurious oscillations, in addition to flux continuity and local conservation.

Due to space limitation, we don't recap the sophisticated control-volume methods in $[15,16]$. But we will test similar numerical examples proposed in the above work, which have strong anisotropy and concentrated sinks and sources.

The mimetic methods $([4,8]$ and the references therein) represent another type noteworthy methods that can be used for Darcy flow calculations. They are developed with intention to incorporate important properties of physical and mathematical models into discrete framework. Somehow WGFEMs echo this idea by introducing new concept of gradients at the discrete (element) level. The mimetic methods are derived from the local consistency condition and use degrees of freedom at vertices, on edges (Gauss-Lobatto points), and inside elements. Similarly, WGFEMs use degrees of freedom on edges and inside elements.

\section{Numerical Experiments}

In this section, we conduct numerical experiments to examine performance of the weak Galerkin finite element methods on solving the Darcy equation. For comparison, we also present numerical results of other commonly used numerical methods, e.g., MFEM and the cell-centered finite difference method on some benchmark problems. Some mesh data structures recommended in the iFEM package [11] are used in our testing code. Some techniques for the Matlab implementations of the lowest order Raviart-Thomas MFEM discussed in [3] are also adopted.

Example 1: A smooth example. Here $\Omega=(0,1)^{2}$, a known exact pressure is specified as $p(x, y)=\sin (2 \pi x) \sin (2 \pi y), \mathbf{K}=\mathbf{I}_{2}$ (the order 2 identity matrix). Accordingly, the source term $f(x, y)=8 \pi^{2} \sin (2 \pi x) \sin (2 \pi y)$. This example has been tested by other researchers $[10,29]$. It has an analytical 
Table 1: Example 1: Convergence rates of the 0th order WGFEMs on triangular and rectangular meshes.

\begin{tabular}{||c||c||c||}
\hline & $\begin{array}{c}\text { Triangular } \\
\left(P_{0}, P_{0}, R T_{0}\right)\end{array}$ & $\begin{array}{c}\text { Rectangular } \\
\left(Q_{0}, P_{0}, R T_{[0]}\right)\end{array}$ \\
\hline$n=1 / h$ & $\left\|Q_{h}^{\circ} p-p_{h}^{\circ}\right\|_{L_{2}}$ & $\left\|p-p_{h}^{\circ}\right\|_{L_{2}}$ \\
\hline 8 & $3.1732 \mathrm{e}-02$ & $1.5844 \mathrm{e}-01$ \\
16 & $8.1927 \mathrm{e}-03$ & $7.9946 \mathrm{e}-02$ \\
32 & $2.0643 \mathrm{e}-03$ & $4.0054 \mathrm{e}-02$ \\
64 & $5.1709 \mathrm{e}-04$ & $2.0037 \mathrm{e}-02$ \\
\hline Conv. rate & 1.98 & 0.99 \\
\hline
\end{tabular}

Table 2: Example 1: Convergence rates of the WGFEM with $\left(P_{1}, P_{1}, R T_{1}\right)$ elements on uniform triangular meshes.

\begin{tabular}{||c||c|c||}
\hline$n=1 / h$ & $\left\|R_{h} \mathbf{u}-\mathbf{u}_{h}\right\|_{L_{2}}$ & $\left\|Q_{h}^{\circ} p-p_{h}^{\circ}\right\|_{L_{2}}$ \\
\hline 8 & $5.0084 \mathrm{e}-02$ & $6.4576 \mathrm{e}-04$ \\
16 & $1.2536 \mathrm{e}-02$ & $8.0296 \mathrm{e}-05$ \\
32 & $3.1440 \mathrm{e}-03$ & $1.0080 \mathrm{e}-05$ \\
64 & $7.8781 \mathrm{e}-04$ & $1.2650 \mathrm{e}-06$ \\
\hline Conv. rate & 1.99 & 2.99 \\
\hline
\end{tabular}

solution for the pressure so that accuracy and convergence rates of errors of various numerical methods can be measured and compared.

Numerical results of the WGFEMs with shape functions in $\left(P_{0}, P_{0}, R T_{0}\right)$, $\left(Q_{0}, P_{0}, R T_{[0]}\right)$, and $\left(P_{1}, P_{1}, R T_{1}\right)$ are tabulated in Tables 1 and 2 . The exact pressure solution is infinitely smooth, so we have $m=\infty$ in Theorem 1 .

In the 2nd column of Table 1, the error between the numerical pressure and the projection of the exact pressure into the constant space has convergence rate 1.98 (close to 2 ). If we measure the error between the numerical pressure and the exact pressure, then the convergence rate should be close to 1 . In the 3rd column of Table 1, the error between the numerical pressure and the exact pressure has convergence rate 0.99 (close to 1 ). If we measure the error between the numerical pressure and the projection of the exact pressure into the constant space, then the convergence rate should be close to 2 . In this regard, the weak Galerkin $\left(P_{0}, P_{0}, R T_{0}\right),\left(Q_{0}, P_{0}, R T_{[0]}\right)$ finite elements behave like the continuous and discontinuous Galerkin linear elements.

In Table 2, Column 2 shows the errors between the numerical Darcy velocity and the projection of the exact Darcy velocity into the $R T_{1}$ space, the convergence rate 1.99 is close to the theoretical estimate 2. Column 3 exhibits the errors between the numerical pressure and the projection of the exact pressure into the $P_{1}$ space, we observe a close to 3 rd order convergence, agreeing well with Theorem 1.

Example 2: An anisotropic permeability. This example is adopted from [16] with minor modifications. In this example, the domain $\Omega=(0,1)^{2}$, 


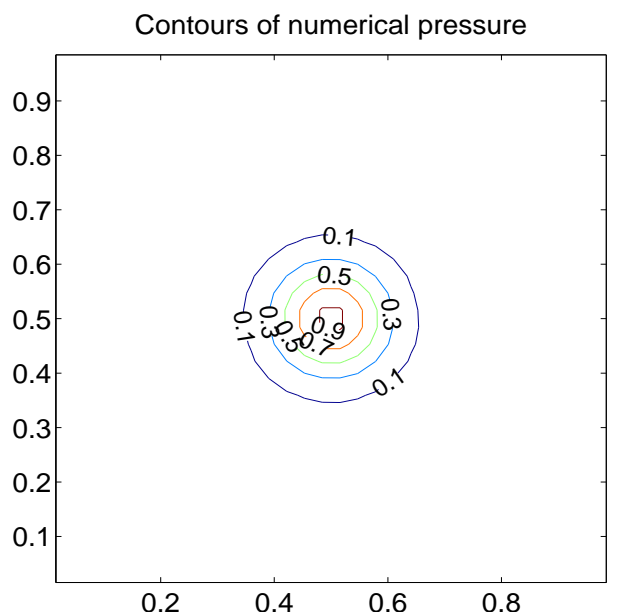

(a) $h=1 / 32$

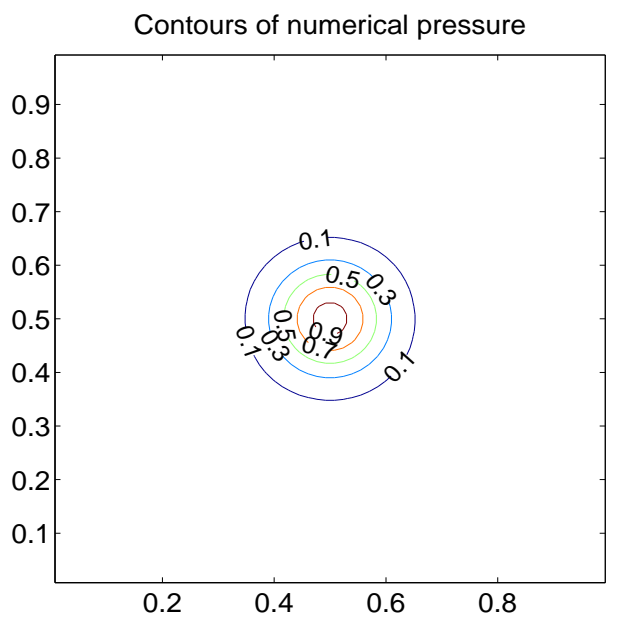

(c) $h=1 / 64$

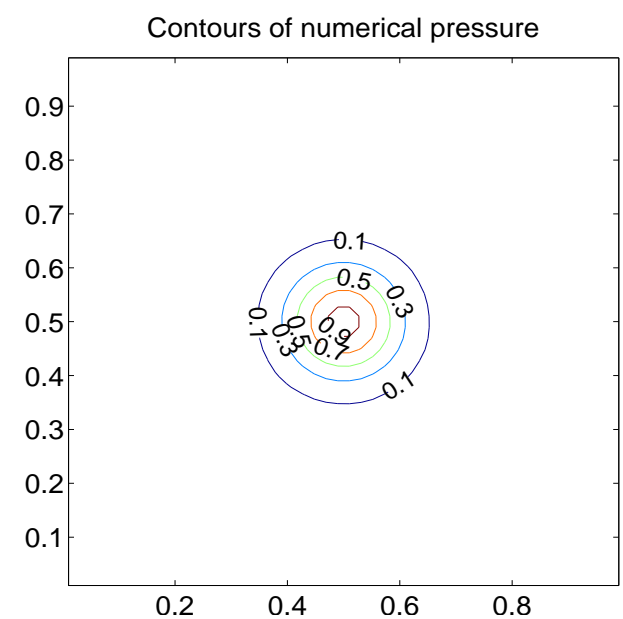

(b) $h=1 / 48$

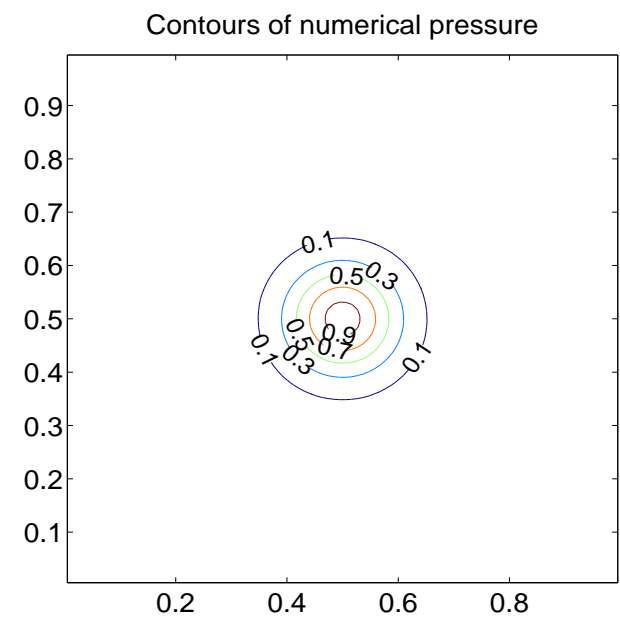

(d) $h=1 / 96$

Figure 3: Example 2: Contour plots of the numerical pressure by using the WGFEM with $\left(Q_{0}, P_{0}, R T_{[0]}\right)$ elements on rectangular meshes. Contour values: $0.1,0.3,0.5,0.7,0.9$.

the permeability tensor

$$
\mathbf{K}=\left[\begin{array}{ll}
a & b \\
b & d
\end{array}\right]=\left[\begin{array}{ll}
0.246436002 & 0.114868364 \\
0.114868364 & 0.053663998
\end{array}\right]
$$

The anisotropy ratio is $3000: 1$ (the ratio of the two positive eigenvalues of the $2 \times 2$ permeability matrix). An exact pressure solution is specified as

$$
p(x, y)=\exp \left(-\frac{\left(x-x_{c}\right)^{2}+\left(y-y_{c}\right)^{2}}{2 \sigma^{2}}\right),
$$


where $\left(x_{c}, y_{c}\right)=(0.5,0.5)$ and $\sigma^{2}=0.005$. Accordingly, the source term is

$$
\begin{gathered}
f(x, y)=\left(\frac{1}{\sigma^{2}}(a+d)-\frac{1}{\sigma^{4}}\left(a\left(x-x_{c}\right)^{2}+2 b\left(x-x_{c}\right)\left(y-y_{c}\right)+d\left(y-y_{c}\right)^{2}\right)\right) \\
\quad \exp \left(-\frac{\left(x-x_{c}\right)^{2}+\left(y-y_{c}\right)^{2}}{2 \sigma^{2}}\right) .
\end{gathered}
$$

As in [16], we examine contours of the numerical pressure to check whether there are any spurious oscillations. We apply the WGFEM with $\left(Q_{0}, P_{0}, R T_{[0]}\right)$ elements on rectangular meshes. The discrete linear systems are solved using the built-in direct solver in Matlab. Shown in Figure 3 are the results for $h=1 / 32,1 / 48,1 / 64,1 / 96$, respectively. Due to the small $\sigma$ value, the pressure is very concentrated. However, it can be observed that the contours of the numerical pressure obtained from the WGFEM are in good shape. There are no obvious oscillations or distortion in the numerical pressure, even though the permeability is strongly anisotropic.

Example 3: A strongly anisotropic discontinuous permeability. This example is similar to the test case 4 in [16]. The test case 4 in [16] is an interesting and challenging example. It has a strongly anisotropic discontinuous permeability and a combination of point source and sinks that are viewed as Green functions.

Here we construct a similar example with specific details so that it can be used for testing of various numerical methods. In this example, the domain $\Omega=(0,1)^{2}$ is divided into four quadrants by the two lines $x=1 / 2$ and $y=1 / 2$. The permeability tensor is

$$
\mathbf{K}_{1}=[2464.36002,1148.68364 ; 1148.68364,536.63998]
$$

in the first and third quadrants, whereas it takes values

$$
\mathbf{K}_{2}=[2464.36002,-1148.68364 ;-1148.68364,536.63998]
$$

in the second and fourth quadrants. Both $\mathbf{K}_{1}$ and $\mathbf{K}_{2}$ are strongly anisotropic, since the ratio of the eigenvalues is $3000: 1$. Furthermore, the eigenvectors of $\mathbf{K}_{1}$ and $\mathbf{K}_{2}$ take different directions and hence generates strong discontinuity in permeability across the dividing lines, see Figure 4 Part (a). A Gaussian source is centered at $\left(x_{c}^{(1)}, y_{c}^{(1)}\right)=(0.25,0.25)$ as

$$
f_{1}(x, y)=10^{5} \exp \left(-\frac{\left(x-x_{c}^{(1)}\right)^{2}+\left(y-y_{c}^{(1)}\right)^{2}}{2 \sigma^{2}}\right),
$$

whereas a Gaussian sink is centered at $\left(x_{c}^{(2)}, y_{c}^{(2)}\right)=(0.75,0.75)$ as

$$
f_{2}(x, y)=-10^{5} \exp \left(-\frac{\left(x-x_{c}^{(2)}\right)^{2}+\left(y-y_{c}^{(2)}\right)^{2}}{2 \sigma^{2}}\right) \text {, }
$$




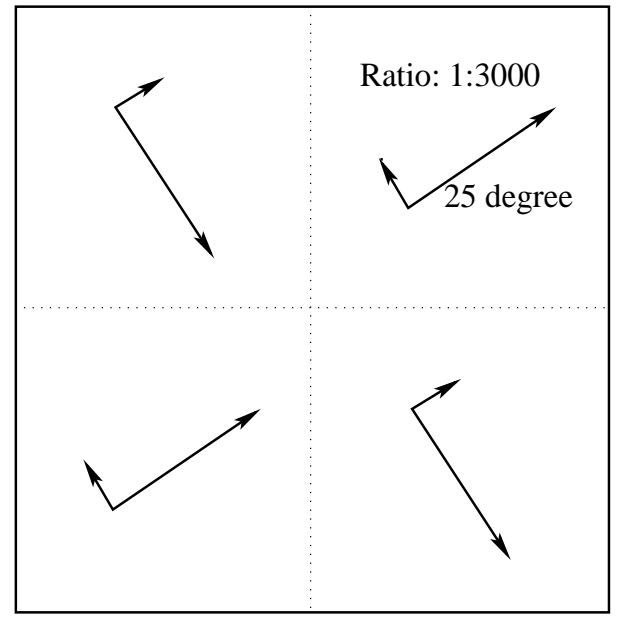

(a) Illustration

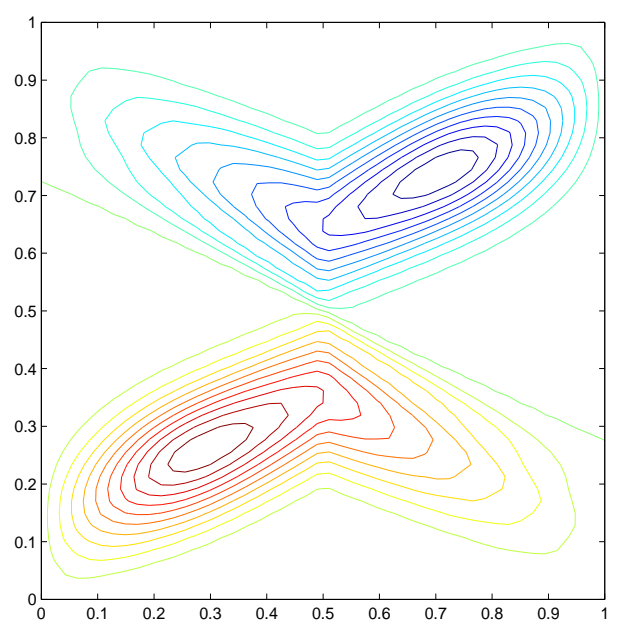

(c) $h=1 / 48$

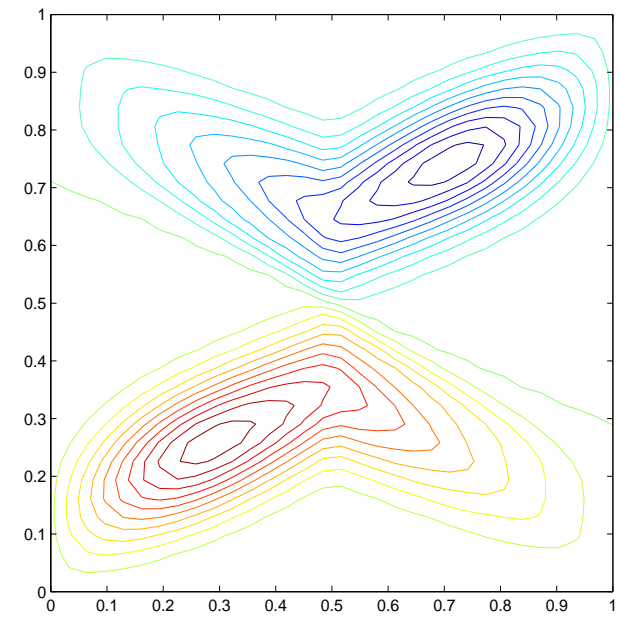

(b) $h=1 / 32$

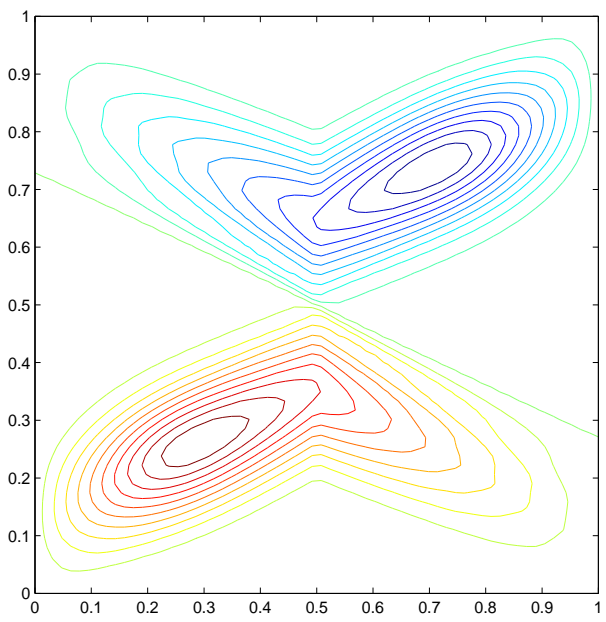

(d) $h=1 / 64$

Figure 4: Example 3: (a) An illustration of the discontinuity and anisotropy in the permeability field. (b), (c), (d) Pressure contours of WG $\left(Q_{0}, P_{0}, R T_{[0]}\right)$ with mesh size $h=1 / 32,1 / 48,1 / 64$ respectively. Contour values vary from -1 to 1 with an increment 0.1

where $\sigma=0.070710$. These Gaussian sink and source are indeed concentrated in very small regions with values varying from near zero to the peak $10^{5}$. Therefore, they could be viewed as approximations to Green functions.

There is no known exact solution for this problem. Shown in Figure 4 are contours of the numerical pressure obtained from applying the WGFEM with $\left(Q_{0}, P_{0}, R T_{[0]}\right)$ elements on rectangular meshes. Parts (b), (c), (d) are respec- 
tively the results with mesh size $h=1 / 32,1 / 48,1 / 64$. The numerical results are already good for $h=1 / 32$, as demonstrated by the shapes and symmetry of the contours. Corresponding to the contour value 0 is a straight line. As the mesh size $h$ decreases, these contours become smoother, especially the inner ones. More importantly, the elliptic shape of these contours reflect the anisotropy in the permeability field. Furthermore, discontinuity in permeability is also reflected in these contours. When contours enter into the 4th quadrant from the 3rd quadrant (or from 1st quadrant into the 2nd quadrant), deflection happens due to the change in the direction of eigenvectors.

Table 3: Example 3: Errors between WG solutions and a CG $P_{2}$ reference solution $p_{\text {ref }}$

\begin{tabular}{||r||c|c||}
\hline$h$ & $\left\|p_{h}^{\circ}-p_{\text {ref }}\right\|_{L_{2}}$ & Conv. rate \\
\hline $1 / 16$ & $7.2840 \mathrm{e}-2$ & 0.98 \\
$1 / 32$ & $3.7415 \mathrm{e}-2$ & 0.96 \\
$1 / 64$ & $2.0076 \mathrm{e}-2$ & 0.90 \\
\hline
\end{tabular}

To further examine the performance of the WGFEM on this example having no known exact solution, we compute the errors between the WG solutions and a reference numerical solution obtained from applying the continuous Galerkin quadratic $\left(P_{2}\right)$ finite elements on a uniform triangular mesh with mesh size 1/512. The close to 1 st order convergence exhibited in Table 3 confirms the improvements in the contours of the WG solutions shown in Figure 4.

Compared to the numerical methods investigated in [16], there is no need for tuning parameters in the WGFEM.

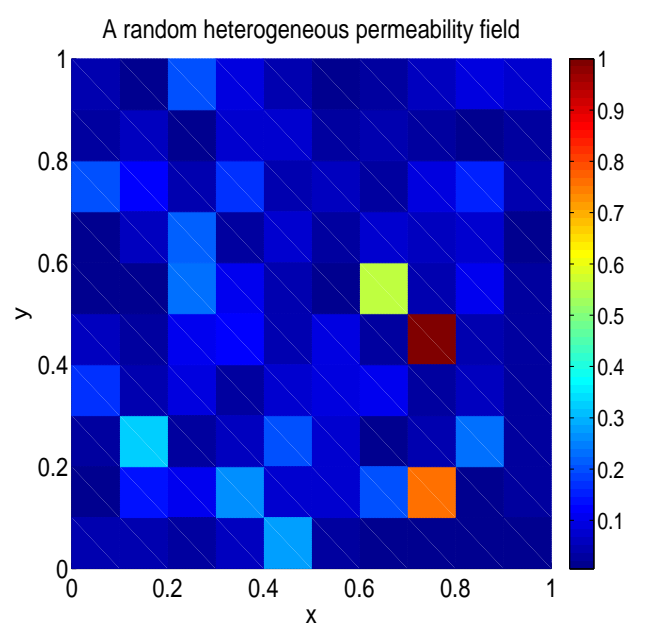

Figure 5: Example 4: A 10x10 random heterogeneous permeability field with $\max =1$, $\min =5.385 \mathrm{E}-3$, ratio $=185.7$

Example 4: A random heterogeneous permeability. Now we con- 
sider an example with a random heterogeneous permeability on the unit square $\Omega=(0,1)^{2}$. The permeability tensor $\mathbf{K}=K_{E} \mathbf{I}_{2}$, where $K_{E}$ is a piecewise constant defined on uniform $10 \times 10$ gridblocks. The value of $K_{E}$ on the rectangular blocks follows a log-normal random distribution. As shown in Figure 5 , the permeability is rescaled so that the maximal value is 1 , the minimal value is $5.385 \times 10^{-3}$, and the heterogeneity ratio (the maximal to the minimal permeability) is 185.7 .

We solve the Darcy equation (1) with the following Dirichlet and Neumann boundary conditions

$$
p=1, \text { left; } \quad p=0 \text {, right; } \quad(-\mathbf{K} \nabla p) \cdot \mathbf{n}=0, \text { top or bottom. }
$$

Similar examples have been studied in $[14,33]$.

No analytical pressure solution is known for this example. Shown in Figure 6 are the simulation results of the numerical pressure and Darcy velocity obtained from using the WGFEM with $\left(Q_{0}, P_{0}, R T_{[0]}\right)$ elements on a $40 x 40$ rectangular mesh. The variation of permeability is clearly reflected in the Darcy velocity profile and contours of the numerical pressure.

It should be pointed out that for this type of problems with piecewise scalar permeability, the WGFEMs and MFEMs with the lowest Raviart-Thomas elements produce numerical results that have negligible differences. This is reflected in the elementwise constant numerical pressures, normal fluxes on the edges, and the total fluxes, see Table 4.

Table 4: Example 4: Total flux: Negligible differences between WG $\left(Q_{0}, P_{0}, R T_{[0]}\right)$ and MFEM $R T_{[0]}$ on rectangular meshes

\begin{tabular}{||r|c|c||}
\hline $1 / h$ & WG $\left(Q_{0}, P_{0}, R T_{[0]}\right)$ total flux & MFEM $R T_{[0]}$ total flux \\
\hline 40 & $5.100392789224447 \mathrm{E}-2$ & $5.100392789226114 \mathrm{E}-2$ \\
50 & $5.120798957711304 \mathrm{E}-2$ & $5.120798957705375 \mathrm{E}-2$ \\
60 & $5.134326628389609 \mathrm{E}-2$ & $5.134326628391268 \mathrm{E}-2$ \\
70 & $5.143966978161513 \mathrm{E}-2$ & $5.143966978159049 \mathrm{E}-2$ \\
80 & $5.151194997447713 \mathrm{E}-2$ & $5.151194997457283 \mathrm{E}-2$ \\
90 & $5.156822170983471 \mathrm{E}-2$ & $5.156822170976758 \mathrm{E}-2$ \\
100 & $5.161332015794227 \mathrm{E}-2$ & $5.161332015787781 \mathrm{E}-2$ \\
\hline
\end{tabular}

For comparison of different numerical methods, we measure the total flux, which is defined as the sum of influxes at the entry $x=0$ or the sum of outfluxes at the exit $x=1$. These two quantities are equal, since there is no sink or source in the domain and there is no flow on the top and bottom boundaries. Shown in Figure 7 are the total fluxes obtained from using the cell-centered finite difference method (CCFDM), the WGFEM with $\left(Q_{0}, P_{0}, R T_{[0]}\right)$ rectangular elements, and the WGFEM with $\left(P_{0}, P_{0}, R T_{0}\right)$ triangular elements. If we use the numerical solution obtained from using CCFDM with 400 partitions in the $x-, y-$ directions as a reference solution, then the total flux is 0.051935 . 

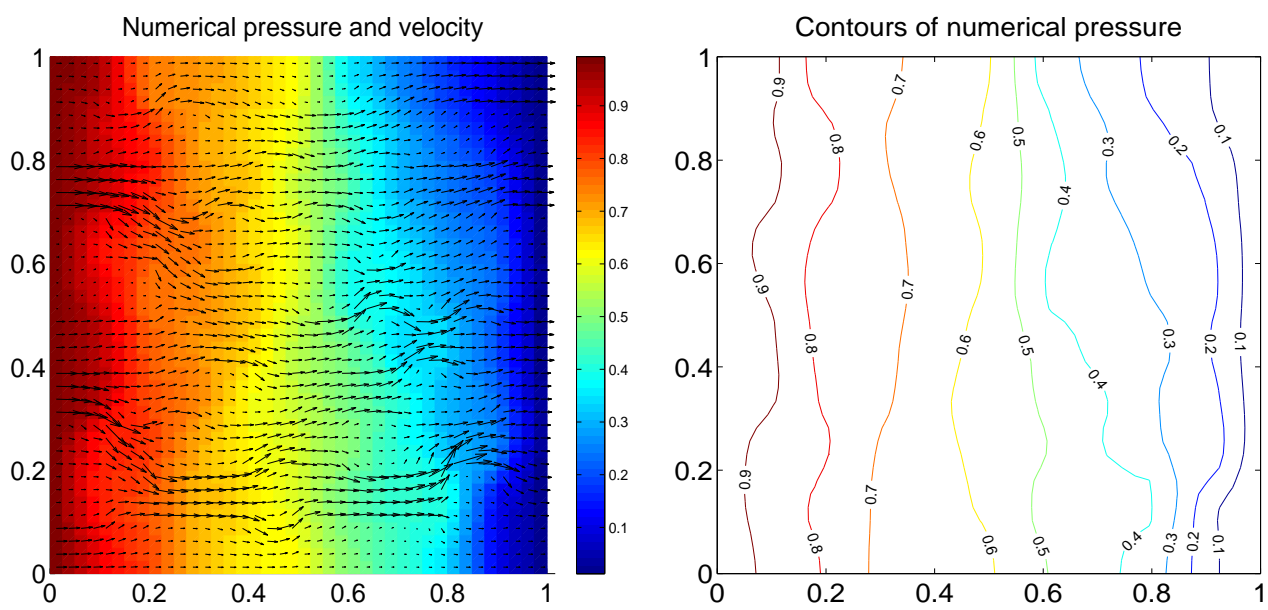

Figure 6: Example 4: Numerical results of WG $\left(Q_{0}, P_{0}, R T_{[0]}\right)$ on a rectangular mesh with mesh size $h=1 / 40$. For better visual effect, the magnitude of the numerical velocity is doubled. Numerical pressure contour values vary from 0.1 to 0.9 with an increment 0.1

The total fluxes are small, because the highest permeability is only 1 and there are many blocks with very low permeability. If the entire domain has permeability one, then the total flux will be 1 , due to the specified boundary conditions.

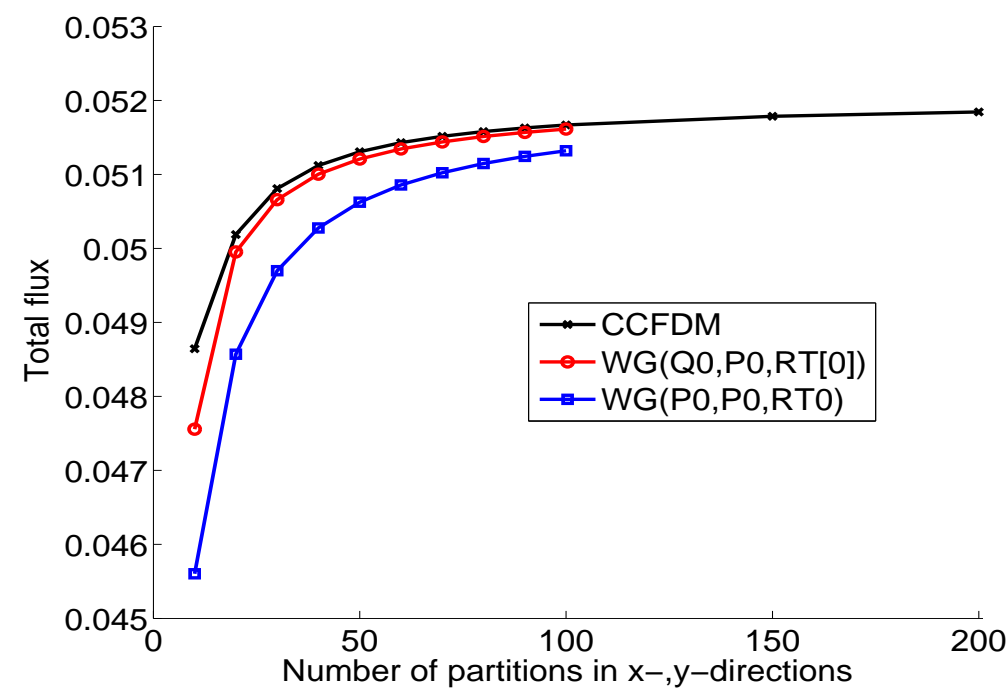

Figure 7: Example 4: The total fluxes of the cell-centered finite difference method (CCFDM), the WGFEM with $\left(Q_{0}, P_{0}, R T_{[0]}\right)$ rectangular elements, and the WGFEM with $\left(P_{0}, P_{0}, R T_{0}\right)$ triangular elements. 
Remark 5.1. Generally speaking, a numerical method for solving (1) involves some type of averages of the permeability over a certain stencil. According to [32], the mixed methods are $\mathbf{K}^{-1}$-methods, whereas the continuous Galerkin methods are $\mathbf{K}$-methods. In this regard, the weak Galerkin methods are $\mathbf{K}$ methods. However, their performances on Example 4 demonstrates "approximation below", a typical feature of $\mathbf{K}^{-1}$-methods.

\section{Concluding Remarks}

It has been observed in our numerical experiments that WGFEMs and MFEMs produce very close numerical results in pressure and flux, when permeability is piecewise constant, even though these two types of methods are established on totally different concepts. There actually exists some "equivalence" between WGFEMs and MFEMs, which can be established via hybridized MFEMs [20].

We examine the equivalence for a simple case $\mathbf{K}=\mathbf{I}_{2}$ :

$$
\nabla \cdot(-\nabla p)=f \text { on } \Omega,\left.\quad p\right|_{\partial \Omega}=0 .
$$

Let $\mathcal{E}_{h}$ be a triangular mesh. Note that the MFEM using $V_{h}=R T_{0}\left(\mathcal{E}_{h}\right) \subset$ $H(\operatorname{div}, \Omega)$ and $W_{h}=P_{0}\left(\mathcal{E}_{h}\right) \subset L^{2}(\Omega)$ seeks $\mathbf{U}_{h} \in V_{h}, P_{h} \in W_{h}$ such that

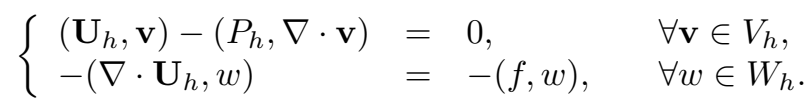

The normal flux continuity built in the definition of $V_{h}=R T_{0}\left(\mathcal{E}_{h}\right)$ can be relaxed through hybridization, i.e., using a space of Lagrange multipliers $M_{h}=P_{0}\left(\Gamma_{h}\right)$ on the edges and a new space $\tilde{V}_{h}=\Pi_{E \in \mathcal{E}_{h}} R T_{0}(E) \supset V_{h}$. This leads to the hybridized MFEM: seek $\tilde{\mathbf{U}}_{h} \in \tilde{V}_{h}, P_{h} \in W_{h}, \lambda_{h} \in M_{h}$ such that

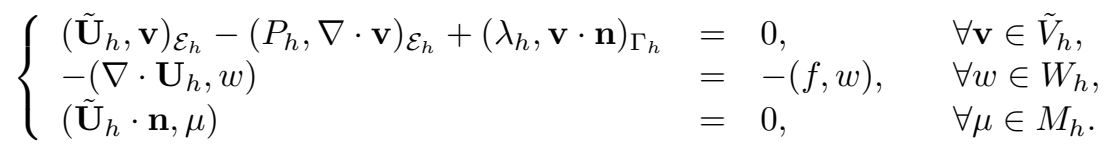

The first equation in (23) can be rewritten elementwise as

$$
\left(-\tilde{\mathbf{U}}_{h}, \mathbf{v}\right)_{E}=\left(\lambda_{h}, \mathbf{v} \cdot \mathbf{n}\right)_{\partial E}-\left(P_{h}, \nabla \cdot \mathbf{v}\right)_{E}, \quad \forall E \in \mathcal{E}_{h} .
$$

If we interpret $\left\{P_{h}, \lambda_{h}\right\}=:\left\{p_{h}^{\circ}, p_{h}^{\partial}\right\}=: p_{h}$ as a discrete weak function, then the right-hand side of the above equation becomes $\left(\nabla_{w, d} p_{h}, \mathbf{v}\right)_{E}$, according to the definition formula (5) for discrete weak gradients. So $-\tilde{\mathbf{U}}_{h}=\nabla_{w, d} p_{h}$, or $\tilde{\mathbf{U}}_{h}=-\mathbf{K} \nabla_{w, d} p_{h}$, since $\mathbf{K}=\mathbf{I}_{2}$ in this case.

This explains the closeness of the numerical solutions of MFEM and WGFEM observed in Example 4. If the space for discrete weak gradients in the WGFEM is chosen as the same vector function space for velocity in the MFEM, then the WGFEM and MFEM have the same degrees of freedom, but the WGFEM 
has less degrees of freedom than that of the hybridized MFEM. Notice that the degrees of freedom for the WGFEM are all for the pressure, whereas the degrees of freedom for the MFEM are for both velocity and pressure.

Of course, this "equivalence" is not a full equivalence. When the source and boundary conditions are nonzero, or the permeability varies spatially, the difference between the WGFEM solution (pressure,flux) and the MFEM solution will be higher order of the mesh size. We shall investigate this further theoretically and numerically.

In this paper, we have investigated the weak Galerkin finite element methods for Darcy flow and demonstrated their potentials as viable tools for numerical simulations of flow in porous media. These novel methods are significantly different than other existing finite element methods. We have observed the following main features of the WGFEMs:

- Locally conservative by design;

- Normal flux is continuous across element interfaces;

- Less or equal number of unknowns (compared to DGFEMs or MFEMs);

- Definite discrete linear systems (compared to MFEMs);

- No need for choosing penalty factors (compared to DGFEMs).

More detailed comparison of the WGFEMs against the DGFEMs and the MFEMs can be found in [22].

From the study presented in this paper, we could conclude that

(i) WGFEMs can adequately handle anisotropy and heterogeneity in Darcy flow;

(ii) WGFEMs can be viable alternatives of the classical MFEMs, when applied to Darcy flow calculations;

(iii) For large-scale computations with Darcy flow, WGFEMs could be more convenient than MFEMs, since the former deal with symmetric definite discrete systems, the latter result in indefinite linear systems.

The Darcy velocity generated by the weak Galerkin methods can be coupled with transport problems. Accurate, efficient, and robust solvers for Darcy flow are much needed for investigating stochastic properties of flow in porous media, especially for three-dimensional numerical simulations. These will be addressed in our future work.

Besides the standard formulations presented in this paper, the weak Galerkin methods also possess mixed and stablized formulations, which can be used as solvers for the Darcy equation as well. The reader is referred to [35].

Acknowledgements: G. Lin would like to acknowledge support by the Applied Mathematics program of the US DOE Office of Advanced Scientific 
Computing Research and Pacific Northwest National Laboratorys Carbon Sequestration Initiative, which is part of the Laboratory Directed Research and Development Program. J. Liu was partially supported by the National Science Foundation under Grant No. DMS-1419077. X. Ye was supported in part by the National Science Foundation under Grant No. DMS-1115097. Computations were performed using the computational resources of Pacific Northwest National Laboratory (PNNL) Institutional Computing cluster systems and the National Energy Research Scientific Computing Center at Lawrence Berkeley National Laboratory. The PNNL is operated by Battelle for the US Department of Energy under Contract DE-AC05-76RL01830.

[1] D.N. Arnold, F. Brezzi, Mixed and nonconforming finite element methods: Implementation, postprocessing and error estimates, Math. Model. Numer. Anal. (M2AN), 19(1985) 7-32.

[2] D.N. Arnold, F. Brezzi, B. Cockburn, L.D. Marini, Unified analysis of discontinuous Galerkin methods for elliptic problems, SIAM J. Numer. Anal., 39(2002) 1749-1779.

[3] C. Bahriawati, C. Carstensen, Three Matlab implementations of the lowestorder Raviart-Thomas MFEM with a posteriori error control, Comput. Meth. Appl. Math, 5(2005) 333-361.

[4] L. Beirao Da Veiga, K. Lipnikov, G. Manzini, Arbitrary-order nodal mimetic discretizations of elliptic problems on polygonal meshes, SIAM J. Numer. Anal., 49(2011) 1737-1760.

[5] M. Benzi, G.H. Golub, J. Liesen, Numerical solution of saddle point problems, Acta Numer., 14(2005) 1-137.

[6] S.C. Brenner, L. Owens, L.Y. Sung, A weakly over-penalized symmetric interior penalty method, Elec. Trans. Numer. Anal. (ETNA), 30(2008) $107-127$.

[7] S.C. Brenner, L.R. Scott, The mathematical theory of finite element methods, Springer, 3rd ed., 2008.

[8] F. Brezzi, A. Buffa, K. Lipnikov, Mimetic finite differences for elliptic problems, Math. Model. Numer. Anal., 43(2009) 277-295.

[9] F. Brezzi, M. Fortin, Mixed and hybrid finite element methods, SpringerVerlag, 1991.

[10] F. Brezzi, T. Hughes, L. Marini, A. Masud, Mixed discontinuous Galerkin methods for Darcy flow, J. Sci. Comput., 22\&23(2005) 119-145.

[11] L. Chen, iFEM: An innovative finite element method package in Matlab.

[12] Z. Chen, G. Huan, Y. Ma, Computational methods for multiphase flows in porous media, SIAM, 2006. 
[13] B. Cockburn, J. Gopalakrishnan, R. Lazarov, Unified hybridization of discontinuous Galerkin, mixed, and continuous Galerkin methods for second order elliptic problems, SIAM J. Numer. Anal., 47(2009) 1319-1365.

[14] L.J. Durlofsky, Accuracy of mixed and control volume finite element approximations to Darcy velocity and related quantities, Water Resour. Res., 30(1994) 965-973.

[15] M.G. Edwards, H. Zheng, Double-families of quasi-positive Darcy-flux approximations with highly anisotropic tensors on structured and unstructured grids, J. Comput. Phys., 229(2010) 594-625.

[16] M.G. Edwards, H. Zheng, A quasi-positive family of continuous Darcyflux finite volume schemes with full pressure support, J. Comput. Phys., $227(2008)$ 9333-9364.

[17] Y. Efendiev, V. Ginting, T. Hou, R.E. Ewing, Accurate multiscale finite element methods for two-phase flow simulations, J. Comput. Phys., 220(2006) $155-174$.

[18] Y. Epshteyn, B. Riviere, Estimation of penalty parameters for symmetric interior penalty Galerkin methods, J. Comput. Appl. Math., 206(2007) 843-872.

[19] R.E. Ewing, The Mathematics of Reservior Simulation, SIAM, 1983.

[20] B. M. Fraejis de Veubeke, Displacement and equilibrium models in the finite element method, In "Stress Analysis", O. Zienkiewicz and G. Holister, eds., Wiley, 1965.

[21] P.A. Forsyth, A control-volume, finite element method for local mesh refinement in thermal reservoir simulation, SPE Reser. Engrg. J., 5(1990) $561-566$.

[22] G. Lin, J. Liu, F. Sadre-Marandi, A comparative study on the weak Galerkin, discontinuous Galerkin, and mixed finite element methods, J. Comput. Appl. Math., 273(2014) 346-362.

[23] G. Lin, A.M. Tartakovsky, An efficient, high-order probabilistic collocation method on sparse grids for three-dimensional flow and solute transport in randomly heterogeneous porous media, Adv. Water Resour., 32(2009) $712-722$.

[24] G. Lin, A.M. Tartakovsky, Numerical studies of three-dimensional stochastic Darcy's equation and stochastic advection-diffusion-dispersion equation, J. Sci. Comput., 43(2010) 92-117.

[25] J. Liu, R. Cali, A note on the approximation properties of the locally divergence-free finite elements, Int. J. Numer. Anal. Model., 5(2008) 693703 . 
[26] J. Liu, L. Mu, X. Ye, L2 Error estimation for DGFEM for elliptic problems with low regularity, Appl. Math. Lett., 25(2012) 1614-1618.

[27] J. Liu, L. Mu, X. Ye, A comparative study of locally conservative numerical methods for Darcy's flows, Procedia Computer Science, 4(2011) 974-983.

[28] L.D. Marini, An inexpensive method for the evaluation of the solution of the lowest order Raviart-Thomas mixed method, SIAM J. Numer. Anal., $22(1985)$ 493-496.

[29] A. Masud, T.J.R. Hughes, A stabilized mixed finite element method for Darcy flow, Comput. Meth. Appl. Mech. Engrg., 191(2002) 4341-4370.

[30] J.E. Morel, R.M. Roberts, M.J. Shashkov, A local support-operators diffusion discretization scheme for quadrilateral meshes, J. Comput. Phys., 144(1998) 17-51.

[31] L. Mu, J. Wang, Y. Wang, X. Ye, A computational study of the weak Galerkin method for second-order elliptic equations, Numer. Alg., 63(2013) 753-777.

[32] T.F. Russell, Relationship among some conservative discretization methods, in: Z. Chen et al. (eds.) "Numerical Treatment of Multiphase Flows in Porous Media", Lec. Notes Phys., Vol. 552, Springer, 2000, 267-282.

[33] S. Sun, J. Liu, A locally conservative finite element method based on piecewise constant enrichment of the continuous Galerkin method, SIAM J. Sci. Comput., 31(2009) 2528-2548.

[34] J. Wang, X. Ye, A weak Galerkin finite element method for second order elliptic problems, J. Comput. Appl. Math., 241(2013) 103-115.

[35] J. Wang, X. Ye, A weak Galerkin mixed finite element method for second order elliptic problems, Math. Comp., 83(2014) 2101-2126. 